\title{
Defining the Skills of Disaster Responders
}

\author{
Elaine Daily, RN, BSN, FCCM, FAHA
}

World Association for Disaster and Emergency Medicine, Madison, Wisconsin USA

Correspondence:

Elaine Daily, RN, BSN, FCCM, FAHA

Consultant, Educator

Madison, Wisconsin 53716 USA

E-mail: elainedaily@gmail.com

\section{Conflicts of interest: none}

Keywords: competency; disaster; skills

Received: September 28, 2016

Accepted: October 16, 2016

Online publication: January 26, 2017

doi:10.1017/S1049023X16001436
Daily E. Defining the skills of disaster responders. Prehosp Disaster Med. 2017;32(2):231-232.

The article "What Kinds of Skills are Necessary for Physicians Involved in International Disaster Response?" by Noguchi et $\mathrm{al}^{1}$ addresses an important issue, particularly as competencies become cornerstones of disaster educational programs. Knowing what specific skills are utilized frequently by disaster responders is key to formulating the competencies to direct appropriate education. I laud the authors for undertaking this inquiry. However, I have some concerns with their methodology as well as their interpretation of results and consequent conclusions.

First of all, although supposedly addressing and evaluating the frequency of performance of the primary skills required for physicians responding to disaster, the survey items were based on nursing skills published in the nursing literature, as well as skills incorporated in the multi-disciplinary Japan Medical Team for Disaster Relief training curriculum. And, indeed, many of the 42 "skills" included in the survey appear more relative to nurses than physicians; although in times of crisis, there is much overlay and parameters become less rigid. Additionally, as no open-ended questions were included in the survey, it is unknown what skills other than the 42 listed might have been performed, or their frequency of performance. I would also argue that the 42 "skills" listed are not actual skills, but rather, activities or arenas of action that require numerous skill sets. Likewise, the authors' categorization of "skills" is open to some question. For example, neither wound irrigation nor the application of a medical dressing are uniquely surgical skills.

The reported finding that "the mean surgical skills score was the highest among all the post-disaster phases" does not appear to be borne out by the data in the table of ranked skills that lists only one surgical skill (wound irrigation or assistance with wound irrigation) in the top 23 skills that were performed "sometimes," "often," or "always." To conclude that "this finding indicates that physicians who participate in international disaster relief need to prepare for surgical procedures" is not supported by the evidence provided.

Finally, the conclusion that "the findings of this study elucidate the primary skill needed for international disaster-relief physicians" is difficult to support as this was not assessed in the study. Although the frequency of some activities or areas of activities in which disasterrelief physicians were involved was enumerated, we are a far cry from articulating an evidence-based set of skills or competencies for disaster-relief physicians or other health care providers.

However, I laud the authors for exploring this important area of study. The disaster health care world is in desperate need of gathering evidence on the functions and skills actually utilized in response efforts. Because we lack such important information, competency-based education remains mired in expert opinions, of which there are many.

I encourage the authors, as well as other investigators, to continue to pursue information that will better inform the development of appropriate competency-based education.

Reference

1. Noguchi N, Inoue S, Shimanoe C, et al. What kinds of skills are necessary for physicians involved in international disaster response? Prehosp Disaster Med. 2016;31(4):397-406. 


\section{Author Reply:}

\section{Norihito Noguchi, RN, PHN, MSN; $;^{1,2}$ Satoshi Inoue, MD, PhD, FACS; ${ }^{3}$ Chisato Shimanoe, PH, PhD; ${ }^{4}$ Kaoru Shibayama, RN, PHN, MSN; ${ }^{1,5}$ Hitomi Matsunaga, RN, PHN, MSN; ${ }^{1}$ Sae Tanaka, RN, PHN, MW ${ }^{1}$ Akina Ishibashi, RN, PHN; ${ }^{1}$ Koichi Shinchi, MD, $\mathrm{PhD}^{1}$}

1. Department of Community and International Health Nursing, Faculty of Medicine, Saga University, Saga, Japan

2. Department of Nursing, Self-Defense Forces Fukuoka Hospital, Fukuoka, Japan

3. Department of Emergency Medicine, Division of Trauma Surgery and Surgical Critical Care, Faculty of Medicine, Saga University, Saga, Japan

4. Department of Preventive Medicine, Faculty of Medicine, Saga University, Saga, Japan

5. Department of Nursing, Saga-Ken Medical Centre Koseikan, Saga, Japan

\section{Correspondence:}

Norihito Noguchi, RN, PHN, MSN

Department of Community and International Health Nursing

Faculty of Medicine, Saga University 5-1-1 Nabeshima, Saga City, Japan 849-8501

E-mail: 13624019@edu.cc.saga-u.ac.jp; alstott40_tampa@yahoo.co.jp
First of all, thank you for being interested in our study. We appreciate your valuable comments and questions in order to further advance our study in this field. We have reached some answers to your inquiries.

Although we searched for previous literature and data concerning primary skills required for physician in an international disaster setting, we only confirmed the nursing literature on the core competency or skills in disaster. That is why 42 skills included in this study were carefully selected from preliminary survey (which we interviewed disaster management experts including seven physicians) and we also showed that 42 skills have a high-validity index and an internal consistency. Please refer to "Measures" section in the original article. ${ }^{1}$

Additionally, it is extremely challenging to recover enough data from open-ended questions, and it is difficult to interpret each answer against open-ended question into a quantifiable scoring system. Although it should be ideal to include all possible skills and competencies which might be require for a disaster site overseas, there are always some limitations in this type of study, as I mentioned in the body of the draft already.

Wound irrigation or the application of a medical dressing may not be specific surgical techniques or skills. However, choice of appropriate type of medical dressings or frequency and actual ways of wound irrigation and subsequent management of total care might be largely dependent upon a surgeon's experience and knowledge. Therefore, we tend to consider them as surgical skills and surgeons should be involved in any wound care.

"The mean surgical skills score was the highest among all the post-disaster phases at more than three weeks following the disaster" was indeed derived from the data in the Table 4. We didn't mean that surgical skills were the most frequent skills among all listed elements. It was very interesting for us to find that surgical skills were highest at more than three weeks after the disaster, which was unexpected for us because the past studies mentioned that surgical skills tended to be required at an early stage of disaster.

As I mentioned earlier, skills listed in this study were elaborately chosen based on previous data and studies which are indeed an evidence-based set of skills and competencies for disaster relief health care providers. We were not sure to what extent the scoring system in this study can actually evaluate appropriate skills and competencies that are required in disaster area. Therefore, it is very important to establish a scoring system based on skills listed in our study which facilitate physicians to choose necessary medical skills at a dispatch site. Further studies will need to explore other aspects of physicians' competencies required at disaster sites, and much more worldwide evidences need to be gathered regarding medical relief activities by physicians in various other kinds of disasters. Researchers are difficult to gather disaster-related data because of ethical concerns and low frequently, so it is inevitable that there are some study limitations in Disaster Medicine.

We will further continue our study to improve a quality of disaster training and education. If Ms. Daily's "Letter to the Editor" is published on PDM, we would like the Editor to publish our reply in order to open dialogue for PDM readers. On behalf of the authors, I would like to thank you for your contribution to and interest in our paper.

Reference

1. Noguchi N, Inoue S, Shimanoe C, et al. What kinds of skills are necessary for physicians involved in international disaster response? Prehosp Disaster Med. 2016;31(4):397-406. 